\title{
EPIDEMIOLOGICAL AND MOLECULAR STUDIES ON RIEMERELLA ANATIPESTIFER INFECTION IN DUCKS
}

\author{
DOHA ABD ALRAHMAN AHMED; MOSTAFA SAIF ELDIN; \\ RAGAB SAYED IBRAHIM AND OMAR AMEN \\ *Department of Avian and Rabbit Diseases, Faculty of Vet. Medicine, Assiut University, Egypt.
}

Received: 23 December 2020; Accepted: 31 December 2020

\begin{abstract}
Infectious serositis is a considerable economic problem in duck industry caused by Riemerella anatipestifer. The current study was conducted to investigate the circulating $R$. anatipestifer in ducks in Assiut Province and assessing their antimicrobial susceptibility. One-hundred and twenty diseased or freshly dead ducks aging 1-18 weeks were examined. Naturally infected birds showed respiratory, nervous, and locomotor disturbances, and low body weight. $R$. anatipestifer was detected in 16.6\% (20) of birds. Among the bacteriologically positive 20 birds, only 10 could be identified by PCR as $R$. anatipestifer with a prevalence rate of $8.33 \%$. The sensitivity biogram revealed that all the obtained isolates were sensitive to amoxicillin, doxycycline, and flumequine while resistance to streptomycin, chloramphenicol, ampicillin, erythromycin, spectinomycin, and cephradine was observed. On the basis of MIC, all isolates had $90-100 \%$ sensitivity to doxycycline and amoxicillin, respectively. Experimentally, the isolated R.anatipestifer strains showed pathogenicity to 14-days-old ducklings.
\end{abstract}

Keywords: Ducks, Riemerella anatipestifer, PCR, MIC, pathogenicity.

\section{INTRODUCTION}

Among the global leading problems confronting duck industry is Riemerella anatipestifer infection that implicates in acute and chronic conditions and can develop into epizootic infectious polyserositis in domestic ducks, mainly young, with a mortality of up to $90 \%$ (Sandhu, 2008; Wang et al., 2014; Majhi et al., 2020). The $R$. anatipestifer is a short to filamentous rod-shaped, Gram-negative, singly or in pairs, non-motile, non-spore-

Corresponding author: Doha Abd Alrahman Ahmed E-mail address: dohabdalrahman780@gmail.com Present address: Department of Avian and Rabbit Diseases, Faculty of Vet. Medicine, Assiut University, Egypt. forming bacterium that is capsulated with Indian ink (Hess et al., 2013; Shancy et al., 2018). Clinically, the infected birds show lethargy, nasal discharge, swollen sinuses, dyspnea, diarrhea and neurologic disturbances (Sandhu, 2003; Wu et al., 2020). Ducklings under 5-weeks old, die within 1 to 2 days after appearance of clinical signs, but the older may survive longer (Deif et al., 2015; Shancy et al., 2018). Fibrinous pericarditis, perihepatitis air-sacculitis, and meningitis with severely congested liver and spleen are the main gross lesions (Sandhu, 2008; Chikuba et al., 2016; Shancy et al., 2018). Conventional microbiological examination is a helping tool in $R$. anatipestifer detection but being time consuming and laborious, recent $R$. anatipestifer specific PCR method is developed revealing great success in fast, 
accurate and reliable identification of the bacteria (Wang et al., 2012; Soman et al., 2014). Antimicrobial agents and improved biosecurity are currently applied to prevent and control $R$. anatipestifer infection in waterfowl farming; however, the increasing resistance to common antibiotics in $R$. anatipestifer seriously challenges the treatment (Chen et al., 2012). Tracing the literature back, only few attempts in Assiut were done by Shahata and Sokkar, (1977); Ibrahim and Shahata, (1991); and Ibrahim and Abd Al-Azeem, (2005) to study this problem in ducks. So this study aimed at investigating incidence of $R$. anatipestifer infection in diseased ducks, and determining the antibacterial susceptibility pattern and pathogenicity of the prevalent $R$. anatipestifer isolates in Assiut Province.

\section{MATERIALS AND METHODS}

\section{Sampling:}

Altogether, 120 specimens (60 livers, 20 lungs and 40 naso-tracheal swabs) from diseased or freshly dead ducks aging 1-18weeks old were collected under complete aseptic condition, over the period from January 2020 to September 2020, from the different diagnostic laboratories in Assiut, Egypt, and transported to the laboratory of Faculty of Veterinary Medicine-Assiut University.

\section{Bacteriological and biochemical examination:}

A methylene blue stained film was examined from each specimen. Swabs from each organ were inoculated separately into trypticase soy broth and incubated at $37^{\circ} \mathrm{C}$ for 24 hours. Then, a loopful from the incubated broth was streaked onto $10 \%$-sheep blood agar (BA) and incubated at $37^{\circ} \mathrm{C}$ under anaerobic conditions for 24 hours. The suspected colonies were sub-cultured into MacConkey agar at $37{ }^{\circ} \mathrm{C}$ for $24 \mathrm{hrs}$. and were stained with gram' stain to be examined microscopically. The purified suspected $R$. anatipestifer colonies were biochemically identified (Indole production,
Urease, Catalase, Oxidase, and Litmus milk tests). Also, sugars (sucrose, glucose, lactose, fructose, maltose, dulcitol, salicin, D-mannitol, galactose) fermentation were tested according to Quinn et al. (2002).

\section{Molecular identification by using Polymerase chain reaction (PCR):}

Purified $R$. anatipestifer genomic DNA was obtained by boiling according to Soman et al. (2014). Briefly, pure colony was suspended in $5 \mathrm{ml}$ of phosphate buffered saline (PBS) and centrifuged at $3000 \times g$ for $10 \mathrm{~min}$ at $4^{\circ} \mathrm{C}$ (repeated thrice until obtaining pellet). The pellet was washed twice in PBS, re-suspended in $100 \mu \mathrm{l}$ of nuclease free water, boiled for $10 \mathrm{~min}$, chilled in ice for $30 \mathrm{~min}$. and centrifuged at $3000 \times g$ for $5 \mathrm{~min}$ at $4^{\circ} \mathrm{C}$. Finally, the supernatant was collected and used as template DNA. $R$. anatipestifer species specific primer set (Forward (F): (5'TTACCGACTGATTGCCTTCTA-3' and Reverse (R): (5'-AGAGGAAGACCGAGG ACATC-3') was used for amplification.

The PCR reaction mixture $(25 \mu l$ total volume) contained 12.5 $\mu \mathrm{l}$ Master mix (One $P C R^{T M}$ master mix (Gene Direx) Code No. MB203-0100), 9.5 $\mu l$ PCR grade water, $1 \mu l$ Forward primer $(10 \mathrm{pM} / \mu \mathrm{L}), 1 \mu l$ Reverse primer $(10 \mathrm{pM} / \mu \mathrm{L}), 1 \mu l$ Template DNA $(25 \mu 1$ total reaction). The reaction was conducted in Veriti thermocycler (Applied biosystems, Germany) following the cycling conditions described by Shancy et al. (2018). Accurately, an initial hot start at $94^{\circ} \mathrm{C}$ for 5 minutes, followed by 35 cycles, each consisting of $95^{\circ} \mathrm{C}$ for 1 minute, $55^{\circ} \mathrm{C}$ for 1 minute, and $72^{\circ} \mathrm{C}$ for 1 minute; and a final extension step at $72^{\circ} \mathrm{C}$ for 4 minutes. The amplified products $(5 \mu \mathrm{l})$ were detected on ethidium bromide-stained $1.5 \%$ agarose gel by visualizing them with UV light in comparison to molecular size of 1001.500bp DNA ladder (RTU, Cat.No.DM001. R500, 11bands). 


\section{Determining the Antibacterial Susceptibility Pattern of $R$. anatipestifer using Standard Disk Diffusion method:}

In-vitro susceptibility of $R$. anatipestifer isolates to 14 antibacterial agents

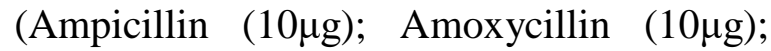
Gentamicin, $(10 \mu \mathrm{g})$; Streptomycin, $(10 \mu \mathrm{g})$; Spectinomycin $(100 \mu \mathrm{g})$; Cephradine $(30 \mu \mathrm{g})$; Erythromycin $(15 \mu \mathrm{g})$; Chloramphenicol

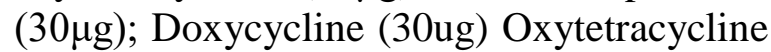
$(30 \mu \mathrm{g}) ; \quad$ sulfa+trimethoprim $(25 \mu \mathrm{g})$ and Flumequine $(30 \mu \mathrm{g}))$ was investigated according to Bauer et al. (1966) following Clinical Laboratory Standards Institute (NCCLS, 1999). CLSI, 2010; and CLSI, 2018).

\section{Detection of minimum inhibitory concentration (MIC):}

The anti-Riemerella effect of Spectinomycin,Streptomycin, Erythromycin, Florphenicol, Sulphaquinoxaline, Doxycycline, Cephradine, Gentamycin, Amoxicillin and Lincomycin was checked in microtiter plate 96 wells using double fold micro-dilution method against all obtained $R$. anatipestifer in a density of $10^{5} \mathrm{CFU}$ (CLSI, 2018). The concentration of each antimicrobial was $10 \mu \mathrm{g} / \mathrm{mL}, 2.56 \mu$ of each antimicrobial was added in 2 wells of the first row of plate then $50 \mu \mathrm{l}$ tryptone soya broth with bacteria was added to all wells. Another $50 \mu \mathrm{l}$ tryptone soya broth with bacteria was added to first row of plate (wells of antimicrobials) then two-fold serial dilution technique was made and discard the last $50 \mu 1$. The bacterial inoculum broth was taken as a positive control and another broth without bacterial inoculum was considered as a negative control. The microtiter plates were incubated at $37^{\circ} \mathrm{C}$ for 24 hours and examined for the lowest concentration showing no detectable growth (MIC).

\section{Pathogenicity testing:}

A total number of 40 one-day-old molar ducks were purchased from (El-Shams Company, Assiut) and reared in clean well ventilated pens. Birds were divided in to two groups (20 per each) and provided with antibiotic-free commercial ration and water ad-libitum. Daily till 14 days old, tracheal swabs were obtained from each group and inoculated in trypticase soy broth for $24 \mathrm{hr}$ and plated on trypticase soy agar to exclude previous Riemerella infection. At day 15, birds in the first group were inoculated intramuscularly with $0.5 \mathrm{ml}$ broth culture containing $10^{6} \mathrm{CFU} / \mathrm{ml}$ of R.anatipestifer isolate .Birds in the second group were treated with sterile broth and kept as negative control.

\section{RESULTS}

Out of the 120 examined ducks, 20 were $R$. anatipestifer infected with a prevalence rate of $16.6 \%$. The most common encountered signs observed in the examined birds were sinusitis, decrease body weight, locomotor disturbances, nervous signs and arthritis. Pericarditis, peri-hepatitis, and airsacculitis were the main postmortem lesions (fig. 1A, B).

\section{Isolation, Bacteriological and biochemical identification:}

Methylene blue stain for tissue smears and blood films of the suspected samples showed typical bipolar cocco-bacillary organisms (fig. 2A, 2B). The produced colonies showed morphological characteristics typical to $R$. anatipestifer on the used culture media (smooth, convex, transparent, glistening, dew drop like, mucoid on the trypticase soy agar and non-hemolytic in blood agar) (fig. 3A, 3B). No bacterial growth was detected on MacConkey agar.

Gram's stained films showed gram-negative coccobacilli that were bipolar in recent cultures. The isolated suspected bacteria showed no evidence of motility on the semisolid agar media. Isolates were urease, catalase and oxidase positive, slow alkaline change of litmus milk. Indole production was negative and could not ferment sugars (glucose, fructose, maltose, sucrose, lactose, Salicin, Dulcitol, and Galactose). 


\section{Molecular characteristics of the obtained isolates:}

Out of the 20 biochemically positive $R$. anatipestifer isolates, 10 isolates produced the typical band of the $R$. anatipestifer specific gene (546 bp) during the molecular examination with a prevalence rate of $8.33 \%$ (fig. 4)

\section{Antimicrobial Susceptibility of the isolated $R$. anatipestifer:}

The isolated $R$. anatipestifer showed in-vitro sensitivity mostly against amoxicillin, doxycycline, and Flumequine and all isolates were absolutely resistant to streptomycin, chloramphenicol, ampicillin, erythromycin, Spectinomycin, and Cephradine (Table 1, Fig. 5).

\section{Minimum inhibitory concentration (MIC):}

On the basis of MIC test for 10 different antimicrobials it was found that all isolates were resistant to 4 antimicrobials (100\%) (Lincomycin, erythromycin, sulphaquinoxaline, Spectinomycin. 9 isolates were resistant to 3antimicrobials (90\%)
(Streptomycin, Cephradine, florphenicol). 9 isolates were sensitive to amoxicillin $(100 \%)$. 9 isolates were sensitive to doxycycline (90\%). 3 isolates were sensitive to gentamicin (30\%). As shown in table (2), table (3) and fig. (6).

\section{Pathogenicity test:}

The ducks aged 14 days were inoculated intramuscularly with $0.5 \mathrm{ml}$ of broth culture containing $10^{6} \mathrm{CFU} / \mathrm{ml}$ of R.anatipestifer isolate NO (1). Clinical picture observed after inoculation was nasal and ocular discharge, mild sinusitis, and congestion of beak, depression, ataxia, ruffled feathers, nervous signs, arthritis and greenish white diarrhea. Two birds died per acutely after 24 hours post inoculation by (I/M route) with septicemic picture (congested lung and enlarged congested liver and spleen). Post 7 days infection, necropsy findings observed as perihepatitis, air sacculitis and pericarditis as shown (fig. 7A\&B, $8 \mathrm{~A} \& \mathrm{~B}, 9 \mathrm{~A} \& \mathrm{~B}, 10$, $11 \mathrm{~A} \& \mathrm{~B} \& \mathrm{C})$. Control group showed No (sings, lesions and deaths). Re isolation of the inoculated organism from experimentally infected ducks was successful.
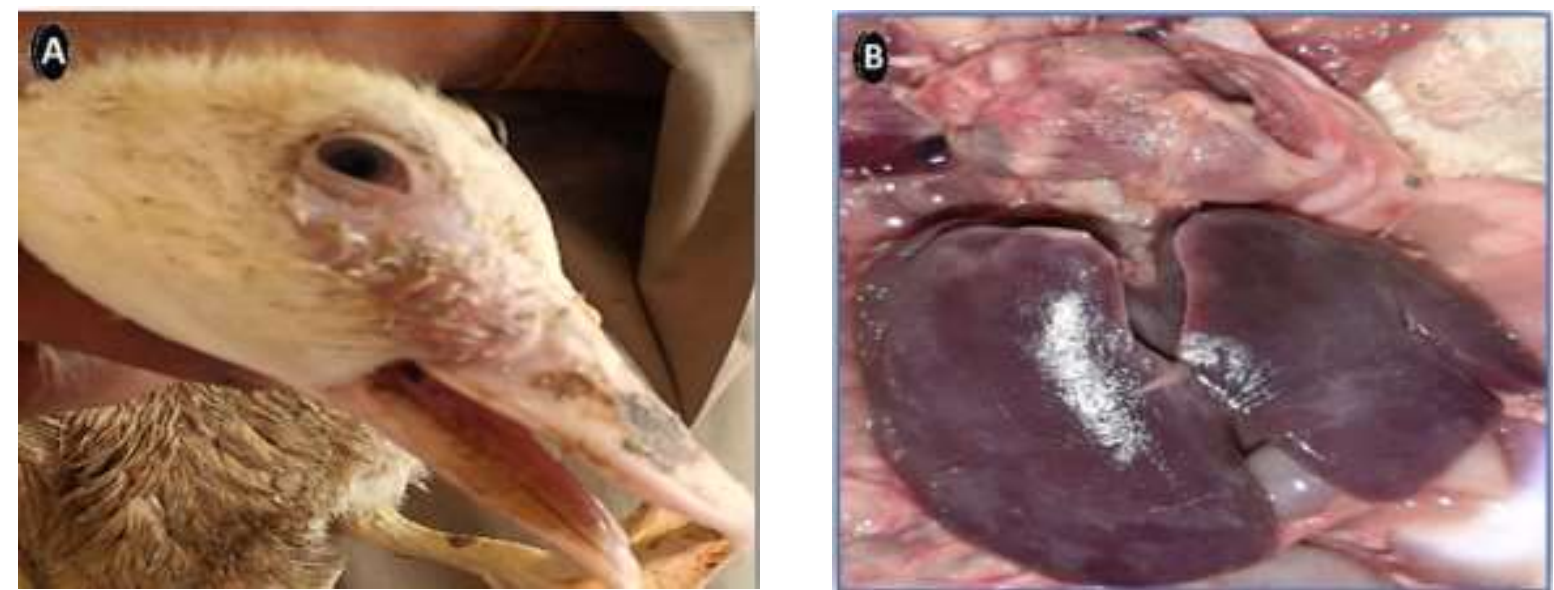

Fig. 1: (A) Sinusitis in naturally $R$. anatipestifer infected ducks. (B) pericarditis, perihepatitis, air sacculitis due to natural infection. 

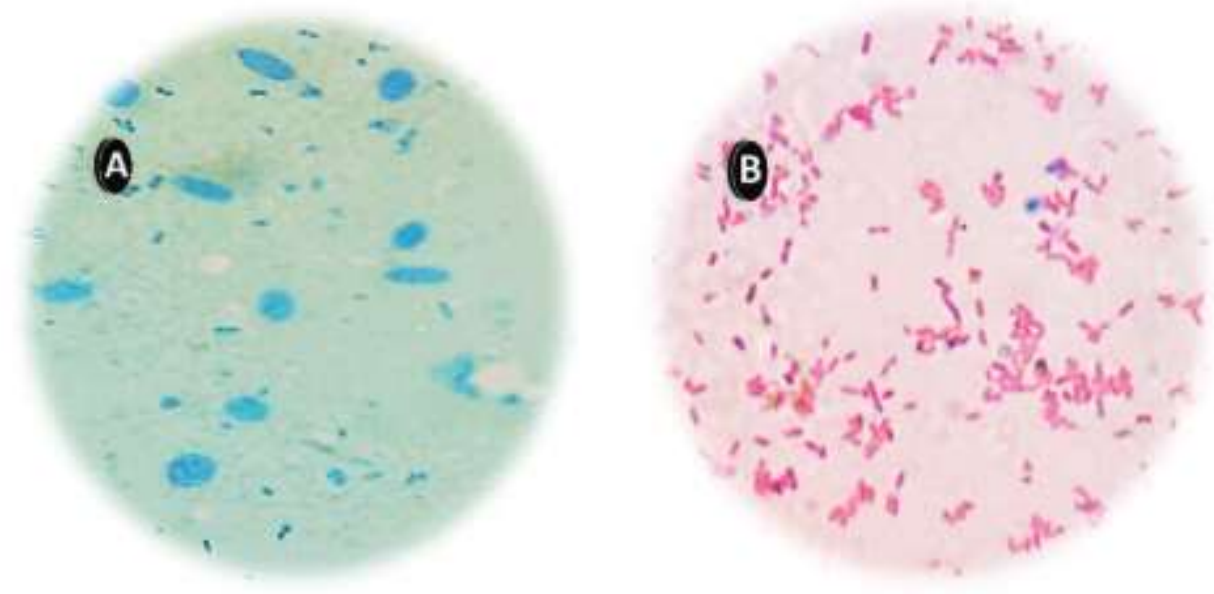

Fig. 2: (A) Methylene blue stained blood smear showing bipolar coccobacilli from $R$. anatipestifer infected duckling. (B) Gram's stained film from a recent culture showing gram negative bipolar cocco-bacilli.
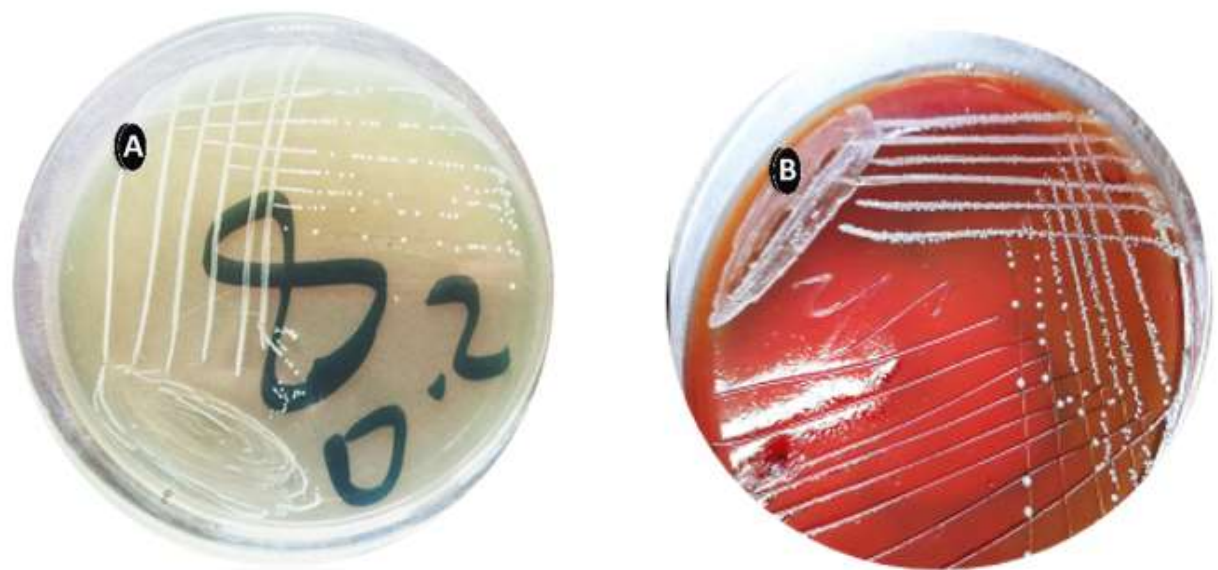

Fig. 3: (A) Dew drop like, mucoid colonies on trypticase soy agar. (B) Dew drop like nonhemolytic colonies on blood agar

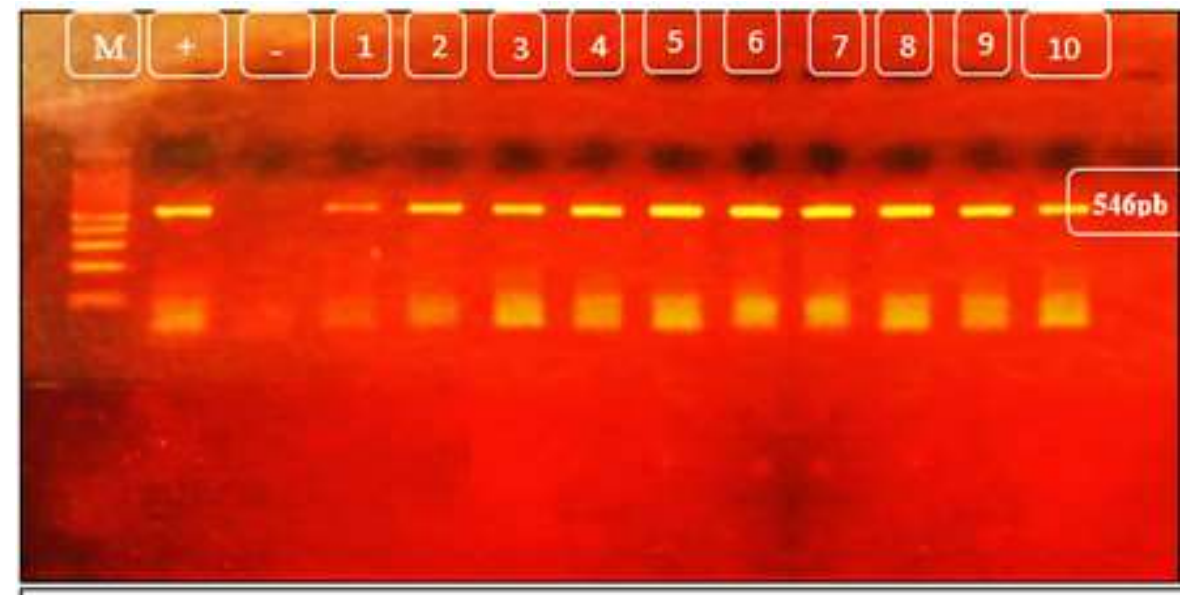

Fig. 4: Agarose gel electrophoresis $1.5 \%$ stained with ethidium bromide showing PCR products of $R$. anatipestifer specific gene illuminate $(546 \mathrm{pb})$ detected in biochemically positive colonies. Lane M: 100 bp ladder as molecular size DNA marker. Lane (+): control positive (pure R. anatipestifer strain). Lane (-): control negative. Lanes 1 to 10: Positive samples. 
Table 1: Antimicrobial sensitivity profile of $R$. anatipestifer isolates.

\begin{tabular}{|l|c|c|c|c|c|c|}
\hline \multirow{2}{*}{ Antibacterial agent } & \multicolumn{2}{|c|}{ Resistant } & \multicolumn{2}{c|}{ Intermediate } & \multicolumn{2}{c|}{ Sensitive } \\
\cline { 2 - 7 } & No & $\%$ & No & $\%$ & No & $\%$ \\
\hline Amoxicillin & 1 & 10 & 3 & 30 & 6 & 60 \\
\hline Ampicillin & 10 & 100 & 0 & 0 & 0 & 0 \\
\hline Doxycycline & 2 & 20 & 3 & 30 & 5 & 50 \\
\hline Oxytetracycline & 9 & 90 & 1 & 10 & 0 & 0 \\
\hline Flumequine & 6 & 60 & 0 & 0 & 4 & 40 \\
\hline Sulfa+trimethoprim & 9 & 90 & 0 & 0 & 1 & 10 \\
\hline Gentamicin & 8 & 80 & 0 & 0 & 2 & 20 \\
\hline Streptomycin & 10 & 100 & 0 & 0 & 0 & 0 \\
\hline Spectinomycin & 10 & 100 & 0 & 0 & 0 & 0 \\
\hline Erythromycin & 10 & 100 & 0 & 0 & 0 & 0 \\
\hline Cephradine & 10 & 100 & 0 & 0 & 0 & 0 \\
\hline Chloramphenicol & 10 & 100 & 0 & 0 & 0 & 0 \\
\hline
\end{tabular}

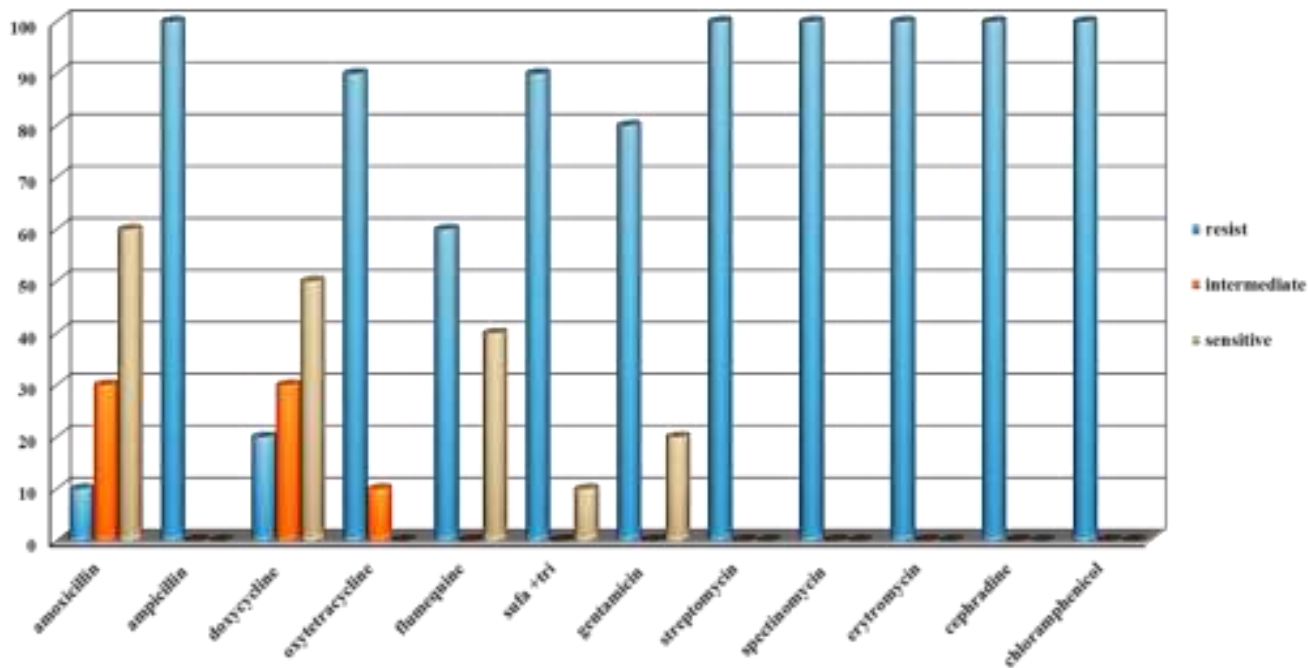

Fig. (5): Antimicrobial sensitivity profile $R$. anatipestifer isolates.

Table 2: MIC of $10 \mathrm{R}$. anatipestifer isolates.

\begin{tabular}{|l|c|c|c|c|c|c|}
\hline \multirow{2}{*}{ Antimicrobial agents } & \multicolumn{2}{|c|}{ Resist } & \multicolumn{2}{c|}{ Intermediate } & \multicolumn{2}{c|}{ sensitive } \\
\cline { 2 - 7 } & No. & $\%$ & No. & $\%$ & No. & $\%$ \\
\hline Amoxicillin & 0 & 0 & 0 & 0 & 10 & 100 \\
\hline Doxycycline & 1 & 10 & 0 & 0 & 9 & 90 \\
\hline Gentamicin & 7 & 70 & 0 & 0 & 3 & 30 \\
\hline florphenicol & 9 & 90 & 0 & 0 & 1 & 10 \\
\hline Streptomycin & 9 & 90 & 0 & 0 & 1 & 10 \\
\hline Cephradine & 9 & 90 & 0 & 0 & 1 & 10 \\
\hline Spectinomycin & 10 & 100 & 0 & 0 & 0 & 0 \\
\hline Lincomycin & 10 & 100 & 0 & 0 & 0 & 0 \\
\hline Erythromycin & 10 & 100 & 0 & 0 & 0 & 0 \\
\hline sulphaquinoxaline & 10 & 100 & 0 & 0 & 0 & 0 \\
\hline
\end{tabular}


Table 3: MIC breakpoint of $R$. anatipestifer isolates.

\begin{tabular}{|l|c|c|c|c|c|c|c|c|c|c|}
\hline $\begin{array}{l}\text { Antimicrobial } \\
\text { agents }\end{array}$ & $<\mathbf{2}$ & $\mathbf{4}$ & $\mathbf{8}$ & $\mathbf{1 6}$ & $\mathbf{3 2}$ & $\mathbf{6 4}$ & $\mathbf{1 2 8}$ & $\mathbf{2 2 5 6}$ & $\begin{array}{c}\text { Resistance } \\
\text { breakpoint }\end{array}$ & $\begin{array}{c}\text { Resistance } \\
\mathbf{\%}\end{array}$ \\
\hline Amoxicillin & 10 & - & - & - & - & - & - & - & $>64$ & $0 \%$ \\
\hline Doxycycline & - & - & 3 & 6 & 1 & - & - & - & $>32$ & $10 \%$ \\
\hline Gentamicin & 2 & - & - & - & 2 & 1 & 2 & 2 & $>16$ & $70 \%$ \\
\hline Florphenicol & - & - & - & - & - & 5 & 4 & - & $>8$ & $90 \%$ \\
\hline Streptomycin & - & 1 & - & - & - & - & 4 & 5 & $>4$ & $90 \%$ \\
\hline Cephradine & - & 1 & - & - & 1 & 3 & 5 & - & $>8$ & $90 \%$ \\
\hline Erythromycin & - & - & - & - & - & - & 5 & 5 & $>8$ & $100 \%$ \\
\hline Lincomycin & - & - & - & - & - & - & - & 10 & $>16$ & $100 \%$ \\
\hline Spectinomycin & - & - & - & - & - & - & 5 & 5 & $>4$ & $100 \%$ \\
\hline Sulphaquinoxaline & - & - & - & - & - & - & - & 10 & $>256$ & $100 \%$ \\
\hline
\end{tabular}

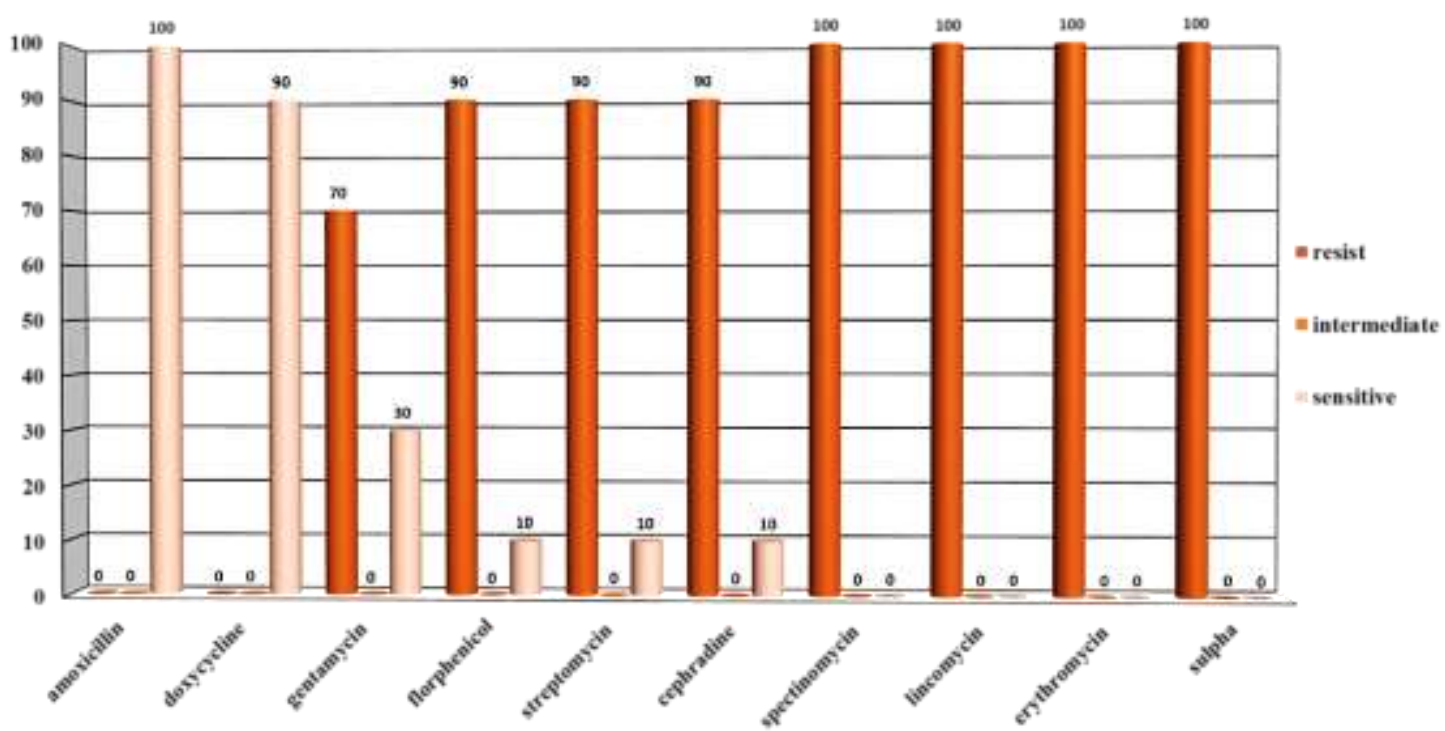

Fig. (6): MIC of $10 R$. anatipestifer isolates.
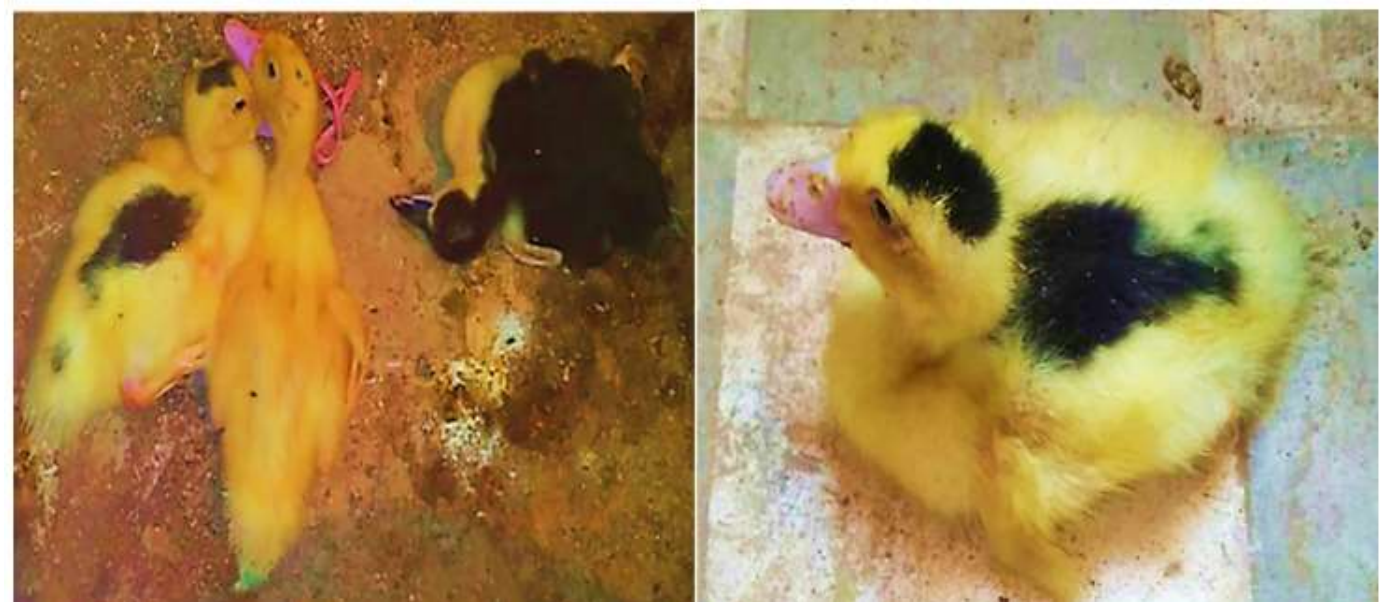

Fig. (7): Experimentally infected ducks show diarrhea, incoordination, ruffled feathers, and anorexia. 


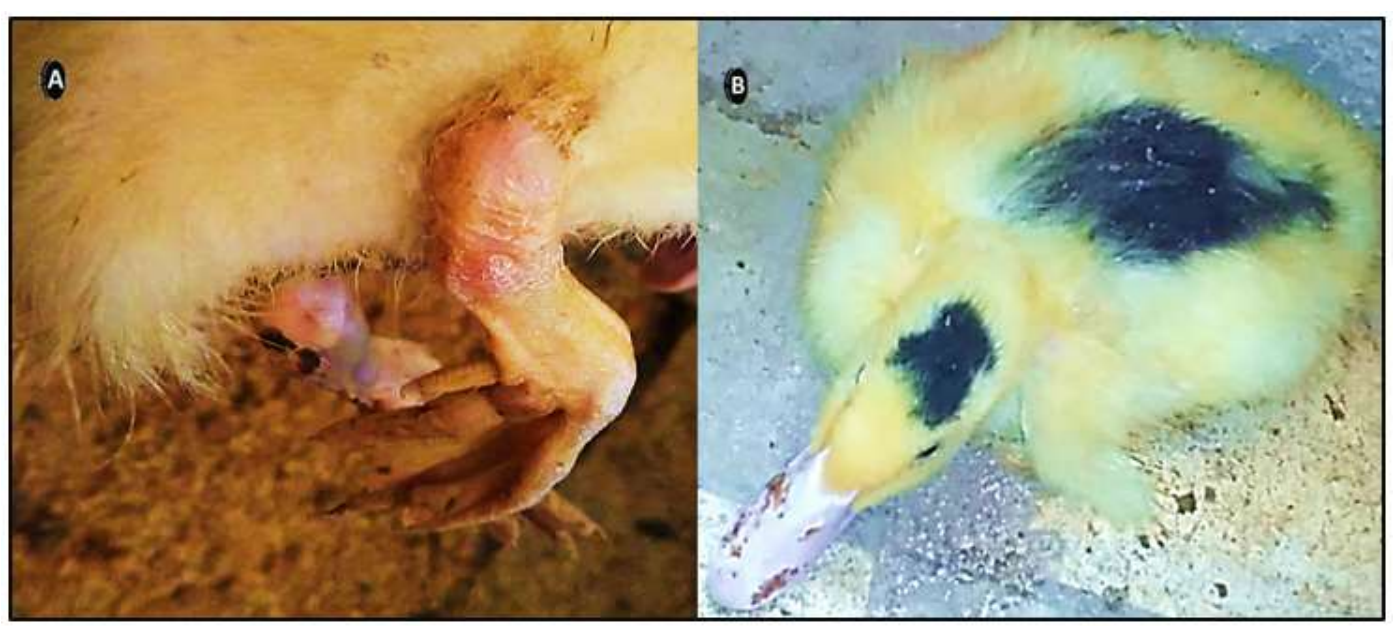

Fig. (8): Experimentally infected ducks show (A) arthritis, and (B) nervous signs

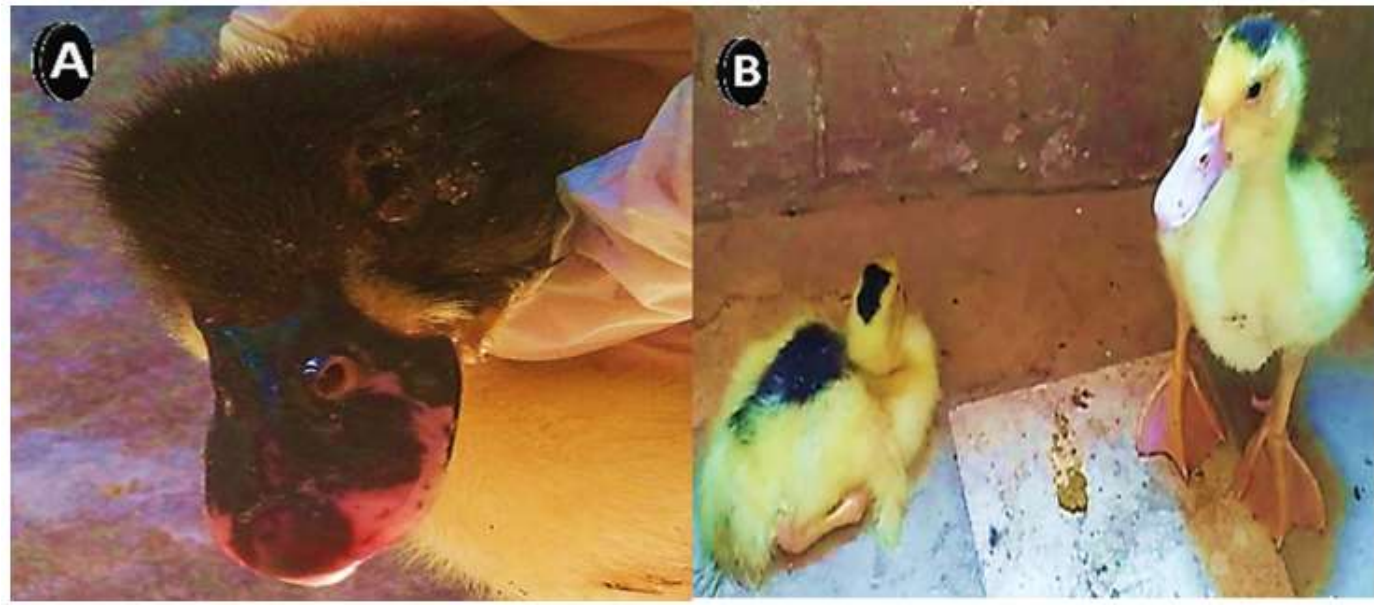

Fig. (9): (A) Experimentally infected ducks show ocular and nasal discharge and congested beak. (B): duck on the right was control and the other on the left was infected duck was infected and show loss of weight

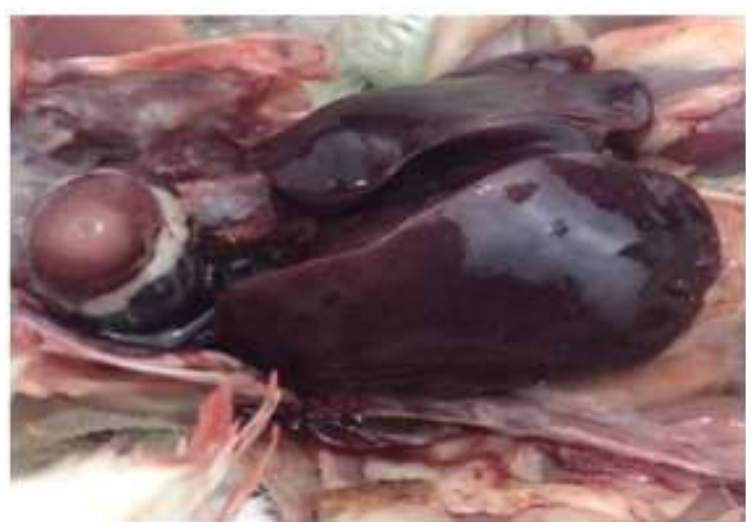

Fig. (10): congested septicemic liver of experimentally infected duck 24hours postinoculation. 


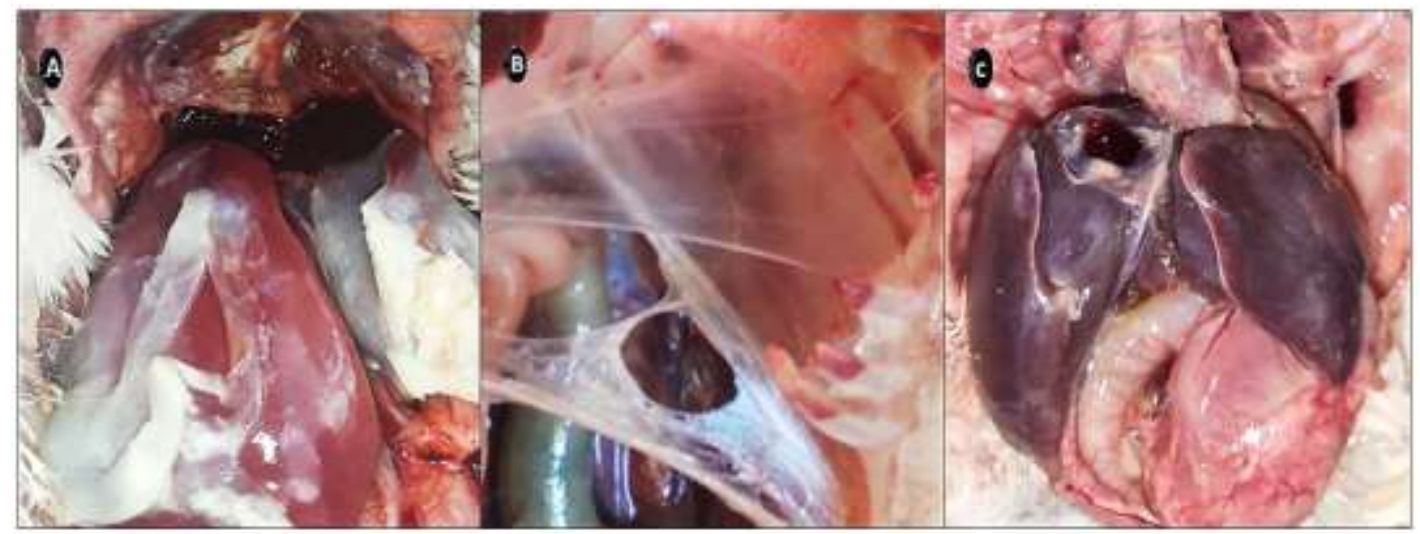

Fig. (11): Illustrates (A) perihepatitis, (B) air sacculitis and (C) pericarditis after 7-days postinoculation of the isolated organism.

\section{DISCUSSION}

$R$. anatipestifer is one of the problems retarding duck industry worldwide causing infectious polyserositis of ducklings, with a mortality up to $90 \%$ (Sandhu, 2008; Wang et al., 2014; Majhi et al., 2020). Our study was designed to determine the prevalence of $R$. anatipestifer infection in ducks and evaluate the antibacterial susceptibility and pathogenicity of the obtained $R$. anatipestifer isolates. In the current work, 20 suspected isolates of the organism were recovered from different duck farms in Assiut Province.

The most common encountered signs observed in the examined birds were sinusitis, decrease body weight, locomotor disturbances, arthritis and nervous signs. These findings were recorded by several authors as Leibovitz, (1972); Leavitt and Ayroud, (1997); Sandhu, (2003); Aparna and Renjith, (2012); Hess et al. (2013) and Wu et al. (2020). Postmortem lesions included lungs congestion, enlarged pinkish liver and enlarged purple spleen, enteritis, congested beak, pericarditis, perihepatitis, airsacculitis, meningitis, osteomyelitis, caseous salpingitis, chronic arthritis and cellulitis. These lesions aligned with those recorded by Aparna and Renjith, (2012).

The produced colonies showed morphological characteristics typical to $R$. anatipestifer on the used culture media. The colonies were smooth, convex, transparent, glistening, dew drop like, mucoid on the trypticase soy agar and non-hemolytic in blood agar, no bacterial growth was detected on macConkey agar. These findings matched with Leavitt and Ayroud, (1997); Shancy et al. (2018) and Majhi et al. (2020). On the other hand some isolates were haemolytic, particularly when plates incubated longer than 48 hrs (Brogden, 1989; Shancy et al., 2018). Among 123 field strains of $R$. anatipestifer, Hinz et al. (1998) recorded that 25 strains showed $\beta$-haemolysis on blood agar after $24 \mathrm{~h}$ to $48 \mathrm{~h}$ incubation.

Concerning cellular morphology of our $R$. anatipestifer isolates, they appear as bipolar cocco-bacillary organisms by methylene blue stain and gram-negative coccobacilli that were bipolar in recent cultures by gram's stain. These results are in agreement with these described by Pillai et al. (1993); Hess et al. (2013); Pala et al. (2013) and Shancy et al. (2018).

In the present study, biochemical tests revealed that $R$. anatipestifer isolates were slow alkaline change of litmus milk; indole production was negative while oxidase, catalase and urease were positive. These findings agree with (Brogden et al., 1982; Soman et al., 2014; Deif et al., 2015 and Surya et al., 2016). The results of sugar fermentation of our isolate revealed that most isolates could not ferment sugars including (glucose, fructose, maltose, 
sucrose, lactose, salicin, dulcitol, and galactose). These findings were also reported by (Brogden et al., 1982 and Bernardet et al., 2002). On the other hand, Priya et al. (2008) and Deif et al. (2015) reported that this bacteria ferments mostly lactose, maltose, dextrose and sucrose.

Bacteriological examination revealed a recovery of $20 R$. anatipestifer isolates with a prevalence rate $16,6 \%$. These results agreed with previous studies reported by Ibrahim and Abd Al-Azeem, (2005). The prevalence rate of $R$. anatipestifer infection ranged from $11 \%-84.4 \% \%$ in ducks worldwide as observed by Huang, (2008); Priya et al. (2008); Wang et al. (2012); Chen et al. (2015) and Majhi et al. (2020). Seventy-six $R$. anatipestifer isolates were detected, and the prevalence in the ducks and geese were $12.3 \%(46 / 375)$ and $8.0 \%$ (30/375), respectively in central Taiwan recorded by Chang et al. (2019). In contrast, Deif et al. (2015) reported that recovery of $20 \mathrm{R}$. anatipestifer isolates with a prevalence rate $16.7 \%$. Out of 69 samples collected from diseased ducks (more than 7 weeks old) revealed isolation of $14 \mathrm{R}$. anatipestifer isolates $(11.7 \%)$. While out of 51 samples collected from diseased ducklings (1-7 weeks old), revealed isolation of 6 isolates $(5 \%)$. Comparing the results obtained from ducks and ducklings showed a higher prevalence rate of ducks $(11.7 \%)$ than ducklings $(5 \%)$.

According to molecular characterization 10 out of $20 R$. anatipestifer isolates with prevalence rate $(8,33 \%)$ were positive in PCR assay which is considered to be a useful laboratory tool for the definitive identification of suspected R. anatipestifer isolates due to absence of selective and/or indicative media for isolation (Rubbenstroth et al, 2009), it was some time difficult to isolate the organism from clinical samples due to overgrowth of other organism. These was described by Higgins et al. (2000) and Cultural and biochemical characteristics based identification of $R$. anatipestifer is time consuming, laborious, and require several days to complete (Soman et al., 2014). Characterization of $R$. anatipestifer by traditional methods is often not sufficient because of phenotypic diversity (Deif et al., 2015). Our results are in disagreement with those described by Wang et al. (2012) who revealed that Using gyrB-PCR to livers of diseased ducks, $46 \%$ riemerellosis incidence rate was recorded in China.

In our recent study, the results of antimicrobial susceptibility test of the isolates showed that the most effective antibiotics were amoxicillin, doxycycline, Flumequine. While all isolates showed absolutely resistant to streptomycin, chloramphenicol, ampicillin, erythromycin, Spectinomycin and Cephradine. Sandhu, (2001) stated that Sulfadimethoxinetrimethoprim was effective in reducing mortality. According to Chang et al. (2003); Zhong et al. (2009) and Deif et al. (2015), all isolates were of high sensitivity to Ciprofloxacin, Norfloxacin, Gentamycin, Chloramphenicol, Polymyxin-B and with moderate sensitivity to Doxycycline, and resistance to penicillin $G$, Metronidazole, Sulfadiazine, Methicillin, Ampicillin, Cefuroxime, Erythromycin. On the basis of MIC test for 10 different antimicrobials it was found that all isolates were resistant to 4antimicrobials (100\%) (Lincomycin, erythromycin, sulphaquinxaline, Spectinomycin. 9 isolates were resistant to 3antimicrobials (90\%) (Streptomycin, Cephradine, florphenicol). All isolates were sensitive to amoxicillin (100\%). Nine isolates were sensitive to doxycycline $(90 \%) .3$ isolates were sensitive to gentamicin $(30 \%)$. Ibrahim and Hussein, (2000) and Ibrahim Abd Al-Azeem, (2005) studied susceptibility of $R$. anatipestifer to different antimicrobials using MIC, they recorded complete susceptibility to penicillin, amoxicillin, enrofloxacin, lincospectin, Oxytetracycline and cephalosporin. Complete resistance to aminoglycosides (streptomycin, gentamycin) and sulfadimethoxin was demonstrated. 
The recorded gross lesions in birds that exposed to the experimental infection with $R$. anatipestifer were pericarditis, perihepatitis, air sacculitis and septicemic lesions especially in liver, spleen and myocardium. Our observation on gross lesions are similar to findings of Tripathy et al. (1980) and Ibrahim and Shahata, (1991) who reported that pericarditis and variable degree of air sacculitis were common lesions, Bayoumi, (1988) who recorded hemorrhage and septicemic picture and Hatfield and Morris, (1988) who observed clinical signs of diarrhea and incoordination in ducklings, severe pericarditis , thickening of air sacs and fibrinous pericarditis in intramuscularly inoculated group of ducklings, Per-acute death with septicemic picture had been seen by Ibrahim and Abd Al-Azeem, (2005). The current results are in disagreement with those described by Asplin, (1956) who said that no clinical signs were observed in any experimental group of duckling.

\section{REFERENCES}

Aparna, S. and Renjith, R. (2012): New duck disease (Riemerella anatipestifer infection) in Animal Husbandry Department, Kerala. J. Ind. Vet. Assoc., Kerala.10 (2).

Asplin, F.D. (1956): A septicemic disease of ducklings Vet. Rec. (67): 854-858.

Bauer, A.W.; W.M.M.; Sherris, J.C. and Turck, M. (1966): Antibiotic Susceptibility test by a standardized single disk method. Am. J Clin. Pathol. 45: 493-496.

Bayoumi, A.H.; Gad, N.; Soliman, A and Atia, M. (1988): "Pasteurella multocida and Pasteurella anatipestifer infection in duck. 1Epidemiological studies". Assiut Vet. Med. J., 20 (40): 87-91.

Bernardet, J.F.; Nagakawa, Y. And Holms, B. (2002): Proposed minimal standards for describing new taxa of the family Flavobacteriaceae and emended description of the family. Int. J. Syst. Evol. Microbiol. (52): 1049-1070.

Brogden, K.A. (1989): "Pasteurella anatipestifer infection. In: Pasteurella and pasteurellosis" p.p:115-139. Academic press limited.

Brogden, K.A.; Rhoades, K.R. and Rimler, R.B. (1982): "serological types and physiologic characteristics of 46 avian Pasteurella anatipestifer cultures" Avian diseases, 26(4):186-190.

Chang, C.F.; Lin, W.H.; Yeh, T.M.; Chiang, T.S. and Chang, Y.F. (2003): Antimicrobial susceptibility of Riemerella anatipestifer isolated from ducks and the efficacy of ceftiofur treatment. J Vet Diagn Invest. (15): 26-29.

Chang, F.F.; Chang, C.C.; Wang, S.H. and Chen, C.L. (2019): Epidemiology and antibiogram of Riemerella anatipestifer isolated from waterfowl slaughterhouses in Taiwan, $\mathrm{J}$ Vet Res (63): 79-86.

Chen, Y.P.; Lee, S.H.; Chou, C.H. and Tsai, H.J. (2012): Detection of florfenicol resistance genes in Riemerella anatipestifer isolated from ducks and geese. Vet Microbiol, (154): 325-331.

Chen, C.L.; Wang, S.T.; Chu, C.; Wang, S.H. (2015): Comparison of four molecular typing methods for Riemerella anatipestifer. Taiwan Vet J. (41):177-185.

Chikuba, T.; Uehara, H.; Fumikura, S.; Takahashi, K.; Suzuki, Y.; Hoshinoo, $K$. and Yamamoto, $Y$. (2016): Riemerella anatipestifer infection in domestic ducks in Japan, 2014. J. Vet. Med. Sci. (78): 16351638.

Clinical and Laboratory Standards Institute (CLSI) (2010): Performance Standards for Antimicrobial Susceptibility Testing: Sixteenth Informational

Supplement M100-S16. CLSI, Wayne, PA, USA. 
Clinical and Laboratory Standards Institute (CLSI) (2018): Performance Standards for Antimicrobial Disk Susceptibility Tests, M100S, 28th Ed., CLSIVol.-38No.3.

Deif, H.; Samir, A.; Mohamed, K.F. and ElJakee, J. (2015): Identifcation of duck septicemia in Egypt. Global Veterinaria (15): 397-400.

Hatfield, R.M. and Morris, B.A. (1988): Influence of the route of infection of Pasteurella anatipestifer on the clinical and immune response of white pekin ducks. Res. Vet. Sci. (44): 208214.

Hess, C.; Enichlmayr, H.; JandreskiCvetkovic, D.; Liebhart, D.; Bilic, I. and Hess, M. (2013): Riemerella anatipestifer outbreaks in commercial goose flocks and identification of isolates by MALDI-TOF mass spectrometry, Avian Pathol, (42): 151156.

Higgins, D.A.; Henry, R.R. and Kounev, Z.V. (2000): Duck immune response to Rimerella antipestifer vaccines. Dev. Comp. Immunol. (24): 153-167.

Hinz, K.H.; Ryll, M.; Köhler, B. and Glünder, G. (1998): Phenotypic characteristics of Riemerella anatipestifer and similar microorganisms from various hosts. Avian Pathol. 27: 33-44.

Huang, C.C. (2008): The natural transmission, serotypes, antimicrobial susceptibility of Riemerella anatipestifer and combinational infection with circoviruses in waterfowl. Department of Veterinary Medicine. National Chiayi University, Chiayi, Taiwan.

Ibrahim, R.S. and Abd AL-Azeem, M.W. (2005): Riemerella anatipestifer infection accounts for major economic losses to meat ducks in Upper Egypt. Assiut Vet. Med. J., 51 (106): 152166.

Ibrahim, R.S. and Hussein, S.Z. (2000): Bacterial agents associated with sinusitis in water fowls and turkeys in
Assiut and El-Menia Governorates. Assiut Vet. Med. J., 44(87): 185-195.

Ibrahim, R.S. and Shahata, M.A. (1991): Some studies on avian pasteurellosis. M. V. Sc., Thesis presented to Dept. Poultry Diseases, Fac. Vet. Med., Assiut University

Leavitt, S. and Ayroud, M. (1997): Riemerella anatipestifer infection of domestic ducklings. Can Vet J (38), 113.

Leibovitz, L. (1972): A survey of the socalled "anatipestifer syndrome." Avian Dis. (16): 836-851.

Majhi, C.; Jena, G.R.; Dash, L.; Kumar, D.; Mishra, S.K.; Mishra, A.; Elmorsy, M.A. and Das, M.R. (2020): Isolation and identification of Riemerella anatipestifer from Duck in Odisha, and its susceptibility to antibiotics and therapeutic management. Journal of Entomology and Zoology Studies, SP8(2): 133-137.

National Committee for Clinical Laboratory Standards (NCCLS) (1999):

Performance Standards for

Antimicrobial Susceptibility Testing. Ninth

Informational Supplement: Approved Standard M100-S9. NCCLS, Wayne, PA.

Pala, S.; Nair, U.R.; Somu, C. and Mahendran, M. (2013): Molecular diagnosis of new duck disease in India by $16 \mathrm{~S}$ rRNA gene based PCR. Adv Anim Vet. Sci., 1(5): 140-142.

Pillai, R.M.; James, P.C.; Punnose, K.T.; Sulochana, S.; Jayaprakasan, V. and Nair, G.K. (1993): Outbreak of pasteurellosis among duck population in Kerala. J. Vet. Anim. Sci., (24): 3439.

Priya, P. M.; Pillai, D. S.; Balusamy, C.; Rameshkumar, $P$. and Senthamilselvan, $P$. (2008): Studies on outbreak of new duck disease in Kerela, India. Int. J. Poult. Sci. (7): 189-190.

Quinn, P. J.; Markey, B.K.; Carter, M.E.; Donnelly, W.J. and Leonard, F.C. (2002): Veterinary Microbiology 
and Microbial Diseases. 1st Edn., Wiley Blackwell Science, USA., 544549.

Rubbenstroth, D.; Ryll, M.; Knobloch, J.K.; Köhler, B. and Rautenschlein, S. (2009): Pathogenesis of Riemerella anatipestifer in turkeys after experimental mono-infection via respiratory routes or dual infection together with the avian metapneumo virus. Avian Pathol, (38): 497-507.

Sandhu, T.S. (2001): Duck health care. International Duck Research Cooperative Inc., pp: 1-6.

Sandhu, T.S. (2003): Riemerella anatipestifer infection. In: Diseases of Poultry, edited by Saif, Y.M.; Barnes, H.J.; Glisson, J.R.; Fadly, A.M.; McDougald, L.R.; Swayne, D.E.: Iowa State Press, Ames, Iowa.. pp: 676-682. Sandhu, T.S. (2008): Riemerella anatipestifer infection. Pages 758-764 in Diseases of Poultry. Y. M. Saif, ed. 12th ed. Blackwell Publishing Ltd., Oxford, UK.

Shancy, C.; Priya, P.M.; Sabnam, V.S.; Radhika, Syam and Mini, M. (2018): RAPID DETECTION OF $R$. anatipestifer ISOLATES USING16SrRNA BASED PCR AND SPECIES- SPECIFIC PCR ASSAY, International Journal of Science, Environment and Technology, 7 :(5): $1802-1812$.

Soman, M.; Nair, S.R.; Mini, M.; Mani, B.K. and Joseph, S. (2014): Isolation and polymerase chain reaction-based identification of Riemerella anatipestifer from duck in kerala, India, Veterinary world (7): 765-769.
Surya, P. S.; Priya, P. M. and Mini, M. (2016): Biotyping and antibiogram of Riemerella anatipestifer from ducks in Kerala. Biosci. Biotech. Res. Comm. 9(3): 457-462.

Tripathy, D.N.; Bangun, A and Hanson, L.E. (1980): "Studies of Pasteurella anatipestifer in ducks". $2^{\text {nd }}$. International symposium of veterinary laboratory diagnosticians, Lucerne, Switzerland: 115-117.

Wang, X.P.; Zhu D.K. .; Wang, M.S.; Cheng, A.C.; Jia, R.Y.; Chen, S.; Chen, X.Y. and Tang, T. (2012): Development and application of specific polymerase chain reaction assay targeting the gyrB gene for rapid detection of Riemerella anatipestifer, Poultry Science (91): 2450-2453.

Wang, X.; Ding, C.; Wang, S.; Han, X.; Hou, W.; Yue, J.; Zou, J. and Yu, S. (2014): The AS87.04050 gene is involved in bacterial lipopolysaccharide biosynthesis and pathogenicity of Riemerella anatipestifer. PLoS One. 9:e109962.

Wu, H.C.; Chang, W.C.; Wu, M.C.; Wang, H.Y. and Chu, C.Y. (2020): Assessment of immunization regimens of duck Riemerella anatipestifer vaccines,Journal of Applied microbiology ISSN. (1): 1185-1192.

Zhong, C.Y.; Cheng, A.C.; Wang, M.S.; Zhu, D.K.; Luo, Q.H.; Chuan, D.Z.; Li, L. and Duan, Z. (2009): Antibiotic susceptibility of Riemerella anatipestifer field isolates. Avian Diseases, (53): 601-607. 


\section{دراسات وبائيه وجزيئيه على الاصابه بميكروب رايميريلا اناتيبيستيفيرفى البط}

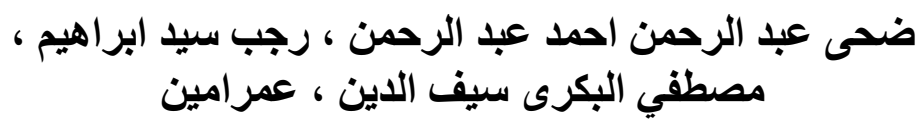

E-mail: dohabdalrahman780@gmail.com

Assiut University web-site: www.aun.edu.eg

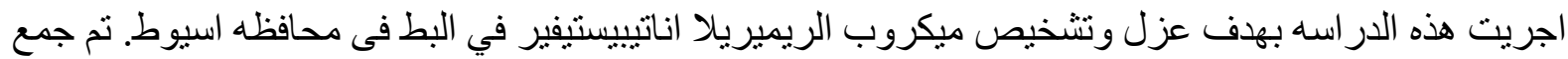

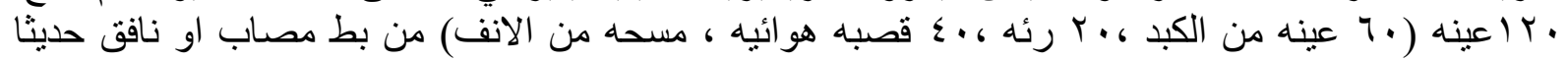

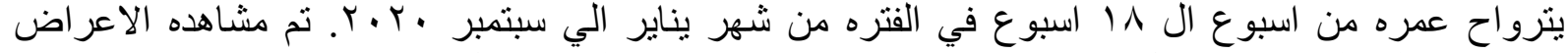

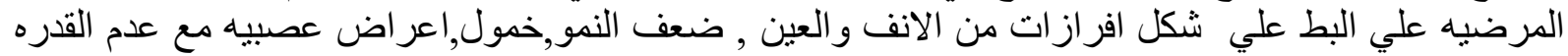

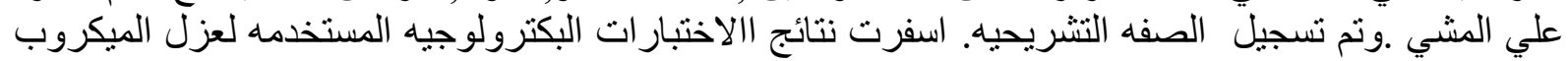

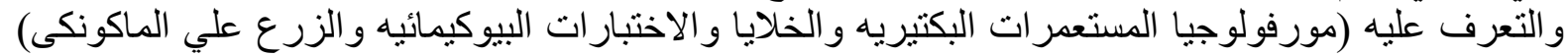

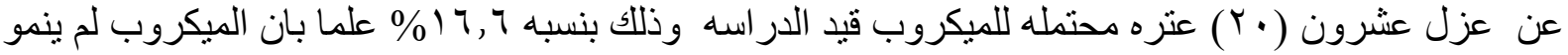

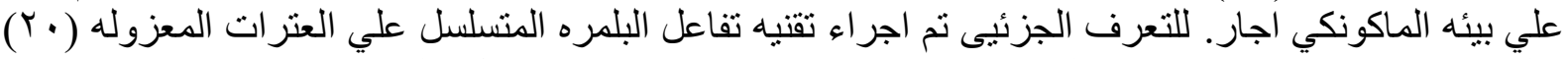

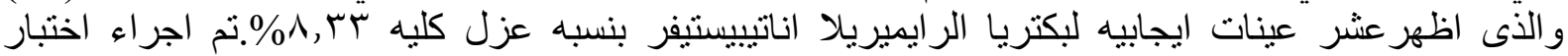

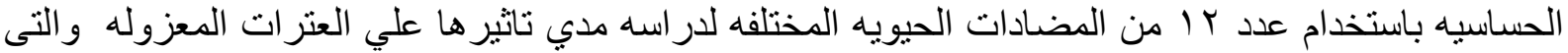

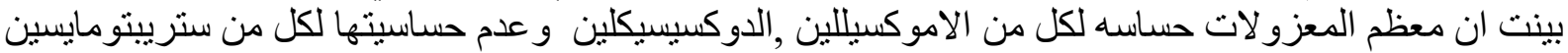

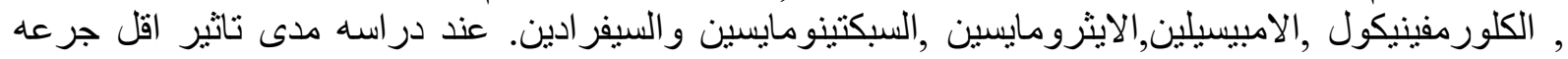

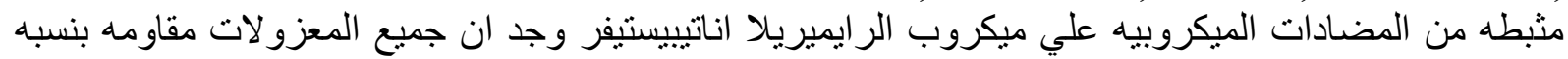

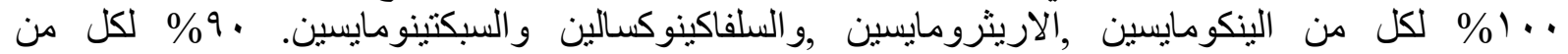

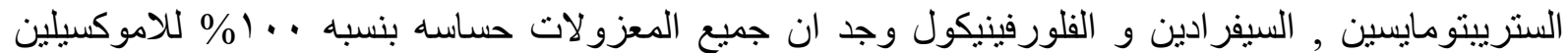

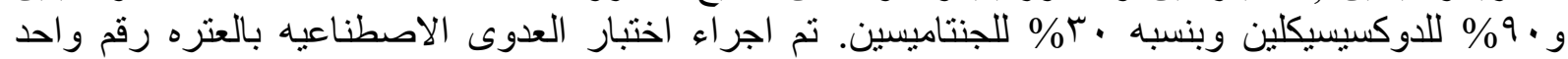
للميكروب المعزول في البط عمر \& ا يوم حقنا في العضل وتم تسجيل الاعر اض الاكلينيكيه و الصفه التشريحيه ونوقشت النتايج . 